\title{
Metabolic disturbances in urinary and plasma samples from seven different systemic autoimmune diseases detected by HPLC-ESI-QTOF-MS
}

Álvaro Fernández-Ochoa ${ }^{1,2, *}$, Carl Brunius ${ }^{3}$, Isabel Borrás-Linares ${ }^{2}$, Rosa Quirantes-Piné2, María de la Luz Cádiz-Gurrea ${ }^{1,2}$, PRECISESADS Clinical Consortium, Marta E. Alarcón Riquelme $e^{4,5, \$}$, Antonio Segura-Carretero ${ }^{1,2, \$}$

${ }^{1}$ Department of Analytical Chemistry, Faculty of Sciences, University of Granada, Av Fuentenueva s/n, Granada 18071, Spain.

${ }^{2}$ Research and Development of Functional Food Centre (CIDAF), Health Science Technological Park, Avda. del Conocimiento, $\mathrm{n}^{\mathrm{o}} 37$, s/n, Granada 18016, Spain.

3 Department of Biology and Biological Engineering, Chalmers University of Technology, SE-412 96 Gothenburg, Sweden.

${ }^{4}$ Centre for Genomics and Oncological Research (GENYO) Pfizer - University of Granada - Andalusian Government, Health Science Technological Park, Av de la Ilustración 114, 18016, Granada, Spain.

${ }^{5}$ Unit of Inflammatory Diseases, Institute of Environmental Medicine, Karolinska Institute, Nobels vag 13, 171 67, Solna, Sweden.

$\star$ These authors share co-senior authorship.

*Author to whom correspondence should be addressed; E-Mail: alvaroferochoa@ugr.es; Department of Analytical Chemistry, Faculty of Sciences, University of Granada, Av Fuentenueva s/n, Granada 18071, Spain.

\section{Supporting Information}

Chemicals

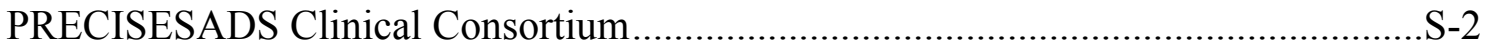

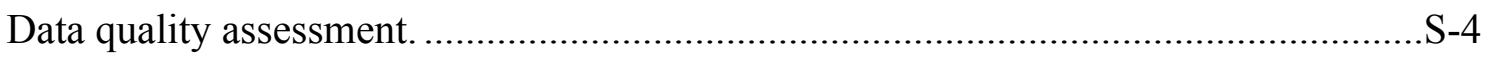

Table S1: Characteristics of the volunteers participating in the study........................... -4

Table S2. Experimental parameters of the LC and MS condition ................................. -5

Table S3. Data processing parameters optimized by IPO ............................................

Table S4. List of metabolites selected in the MUVR-PLS models that compared all

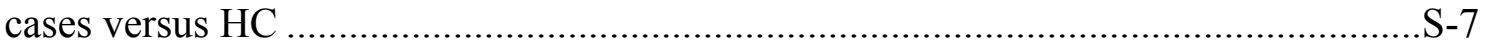

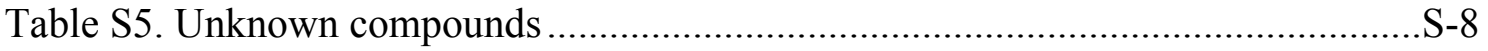

Table S6. ANOVA results for the top six metabolites............................................... -9

Table S7. Annotated metabolites selected from the PLS models (individual SADs

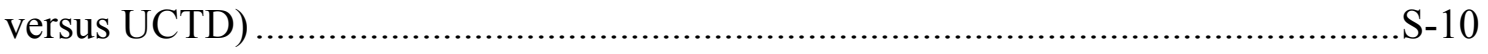

Figure S1. PCA scores plots before and after normalization (color code by batch)....S-11

Figure S2. PCA scores plots before and after normalization (color by class) .............S-12

Figure S3. Results of the MUVR-PLS models of specific SADs versus HC..............S-12 


\section{Chemicals}

All chemicals were of analytical reagent grade and used as received. Formic acid and LC-MS grade methanol for analytical chromatography were purchased from Fluka, Sigma-Aldrich (Steinheim, Germany) and Fisher Scientific (Madrid, Spain), respectively. Water was purified by a Milli-Q system from Millipore (Bedford, MA, USA). For plasma treatment, ethanol and methanol (Fisher Scientific Madrid, Spain) were used.

\section{PRECISESADS Clinical Consortium}

\begin{tabular}{|c|c|}
\hline Researchers and clinicians & Clinical center \\
\hline Lorenzo Beretta, Barbara Vigone & $\begin{array}{l}\text { Referral Center for Systemic Autoimmune } \\
\text { Diseases, Fondazione IRCCS Ca' Granda } \\
\text { Ospedale Maggiore Policlinico di Milano, Italy. }\end{array}$ \\
\hline $\begin{array}{c}\text { Jacques-Olivier Pers, Alain Saraux, } \\
\text { Valérie Devauchelle-Pensec, Divi } \\
\text { Cornec, Sandrine Jousse-Joulin }\end{array}$ & $\begin{array}{l}\text { Centre Hospitalier Universitaire de Brest, } \\
\text { Hospital de la Cavale Blanche, Brest, France. }\end{array}$ \\
\hline $\begin{array}{c}\text { Bernard Lauwerys, Julie Ducreux, } \\
\text { Anne-Lise Maudoux }\end{array}$ & $\begin{array}{l}\text { Pôle de pathologies rhumatismales } \\
\text { systémiques et inflammatoires, Institut de } \\
\text { Recherche Expérimentale et Clinique, } \\
\text { Université catholique de Louvain, Brussels, } \\
\text { Belgium. }\end{array}$ \\
\hline $\begin{array}{c}\text { Carlos Vasconcelos, Ana Tavares, } \\
\text { Esmeralda Neves, Raquel Faria, } \\
\text { Mariana Brandão }\end{array}$ & Centro Hospitalar do Porto, Portugal. \\
\hline $\begin{array}{l}\text { Ana Campar, António Marinho, } \\
\text { Fátima Farinha, Isabel Almeida }\end{array}$ & $\begin{array}{l}\text { Servicio Cantabro de Salud, Hospital } \\
\text { Universitario Marqués de Valdecilla, Santander, } \\
\text { Spain. }\end{array}$ \\
\hline $\begin{array}{l}\text { Miguel Angel Gonzalez-Gay } \\
\text { Mantecón, Ricardo Blanco Alonso, } \\
\text { Alfonso Corrales Martínez }\end{array}$ & $\begin{array}{l}\text { Servicio Cantabro de Salud, Hospital } \\
\text { Universitario Marqués de Valdecilla, Santander, } \\
\text { Spain. }\end{array}$ \\
\hline $\begin{array}{l}\text { Ricard Cervera, Ignasi Rodríguez- } \\
\text { Pintó, Gerard Espinosa }\end{array}$ & $\begin{array}{l}\text { Hospital Clinic I Provicia, Institut } \\
\text { d'Investigacions Biomèdiques August Pi i Sunyer, } \\
\text { Barcelona, Spain. }\end{array}$ \\
\hline Rik Lories, Ellen De Langhe & Katholieke Universiteit Leuven, Belgium. \\
\hline Nicolas Hunzelmann, Doreen Belz & Klinikum der Universitaet zu Koeln, Cologne, \\
\hline
\end{tabular}




\begin{tabular}{|c|c|}
\hline & Germany. \\
\hline Torsten Witte, Niklas Baerlecken & Medizinische Hochschule Hannover, Germany. \\
\hline $\begin{array}{c}\text { Georg Stummvoll, Michael Zauner, } \\
\text { Michaela Lehner }\end{array}$ & Medical University Vienna, Vienna, Austria. \\
\hline $\begin{array}{c}\text { Eduardo Collantes, Rafaela Ortega- } \\
\text { Castro, } \mathrm{M}^{\mathrm{a}} \text { Angeles Aguirre- } \\
\text { Zamorano, Alejandro Escudero- } \\
\text { Contreras, } \mathrm{M}^{\mathrm{a}} \text { Carmen Castro- } \\
\text { Villegas }\end{array}$ & $\begin{array}{l}\text { Servicio Andaluz de Salud, Hospital Universitario } \\
\text { Reina Sofía Córdoba, Spain. }\end{array}$ \\
\hline $\begin{array}{c}\text { Norberto Ortego, María Concepción } \\
\text { Fernández Roldán }\end{array}$ & $\begin{array}{l}\text { Servicio Andaluz de Salud, Complejo hospitalario } \\
\text { Universitario de Granada (Hospital Universitario } \\
\text { San Cecilio), Spain. }\end{array}$ \\
\hline $\begin{array}{c}\text { Enrique Raya, Inmaculada Jiménez } \\
\text { Moleón }\end{array}$ & $\begin{array}{l}\text { Servicio Andaluz de Salud, Complejo hospitalario } \\
\text { Universitario de Granada (Hospital Virgen de las } \\
\text { Nieves), Spain. }\end{array}$ \\
\hline $\begin{array}{l}\text { Enrique de Ramon, Isabel Díaz } \\
\text { Quintero }\end{array}$ & $\begin{array}{l}\text { Servicio Andaluz de Salud, Hospital Regional } \\
\text { Universitario de Málaga, Spain }\end{array}$ \\
\hline $\begin{array}{l}\text { Pier Luigi Meroni, Maria Gerosa, } \\
\text { Tommaso Schioppo, Carolina Artusi, }\end{array}$ & Università degli studi di Milano, Milan, Italy. \\
\hline $\begin{array}{l}\text { Carlo Chizzolini, Aleksandra Zuber, } \\
\text { Donatienne Wynar, }\end{array}$ & Hospitaux Universitaires de Genève, Switzerland. \\
\hline $\begin{array}{l}\text { Laszló Kovács, Attila Balog, } \\
\text { Magdolna Deák, Márta Bocskai, } \\
\text { Sonja Dulic, Gabriella Kádár }\end{array}$ & University of Szeged, Szeged, Hungary. \\
\hline Falk Hiepe, Velia Gerl, Silvia Thiel & Charite, Berlin, Germany. \\
\hline $\begin{array}{c}\text { Manuel Rodriguez Maresca, Antonio } \\
\text { López-Berrio, Rocío Aguilar- } \\
\text { Quesada, Héctor Navarro-Linares }\end{array}$ & $\begin{array}{l}\text { Andalusian Public Health System Biobank, } \\
\text { Granada, Spain }\end{array}$ \\
\hline $\begin{array}{l}\text { Yiannis Ioannou, Chris Chamberlain, } \\
\text { Jacqueline Marovac. }\end{array}$ & $\begin{array}{l}\text { UCB Pharma, Slough, United Kingdom } \\
\text { (PRECISESADS Project office) }\end{array}$ \\
\hline $\begin{array}{l}\text { Marta Alarcón Riquelme, Tania } \\
\text { Gomes Anjos. }\end{array}$ & $\begin{array}{l}\text { Department of Medical Genomics, Center for } \\
\text { Genomics and Oncological Research (GENYO), } \\
\text { Granada, Spain (PRECISESADS Project Office) }\end{array}$ \\
\hline
\end{tabular}




\section{Data quality assessment.}

Before statistical analysis, the quality of the data derived from the metabolomic analysis was evaluated based on the distribution of the QC samples in the PCA analyses. Figure 1S(a-b) shows the results of the PCA scores obtained with the raw data from urine and plasma samples. As shown in the figure, large batch effects are clearly observed affecting sample distributions in both types of biological samples. These batch effects were corrected after normalization using batchCorr package, which takes into account different possible drift trends for each metabolite along the analytical sequences [26]. The PCA scores plots obtained with the normalized data showed the correction of the batch effects (Figure 1S, c-d) demonstrating, therefore, the good efficiency of the normalization process as well as the good quality of the data used for the statistical analyses. Finally, no outliers were detected when PCA was performed with the final data without the QC samples. However, the clusters between the different categories of the samples were not observed in the PCA scores plots (Figure 2S). The biological variability across individuals together with the fact that SADs are closely related produce extremely complex metabolomic matrices. Therefore, unsupervised methods based on PCA were not enough to separate samples based on the different disease diagnoses.

Table S1: Characteristics of the volunteers participating in the study.

Characteristics of the volunteers participating in the study (age, gender, duration of the diseases).

\begin{tabular}{|c|c|c|c|c|c|c|c|c|}
\hline & HC & RA & SLE & SjS & SSc & UCTD & MCTD & PAPS \\
\hline Subjects & 55 & 47 & 46 & 46 & 43 & 22 & 14 & 10 \\
\hline Age & $44.4 \pm$ & $59.2 \pm$ & $48.0 \pm$ & $57.1 \pm$ & $61.1 \pm$ & $52.5 \pm$ & $51.7 \pm$ & $43.4 \pm$ \\
& 12.0 & 11.5 & 11.4 & 12.3 & 10.5 & 11.3 & 16.6 & 14.2 \\
\hline Females, & 44 & 35 & 44 & 44 & 36 & 21 & 13 & 9 \\
n (\%) & $(80.0)$ & $(74.4)$ & $(95.6)$ & $(95.6)$ & $(83.7)$ & $(95.5)$ & $(92.8)$ & $(90.0)$ \\
\hline Duration & -- & $13.6 \pm$ & $13.9 \pm$ & $9.2 \pm$ & $12.2 \pm$ & $9.4 \pm$ & $9.6 \pm$ & $8.8 \pm$ \\
(year) & & 8.4 & 9.4 & 5.5 & 10.2 & 10.9 & 7.2 & 7.3 \\
\hline
\end{tabular}


Table S2. Experimental parameters of the LC and MS condition

Experimental parameters of the liquid chromatographic and mass spectrometry conditions for the untargeted metabolomics analyses of plasma and urine samples.

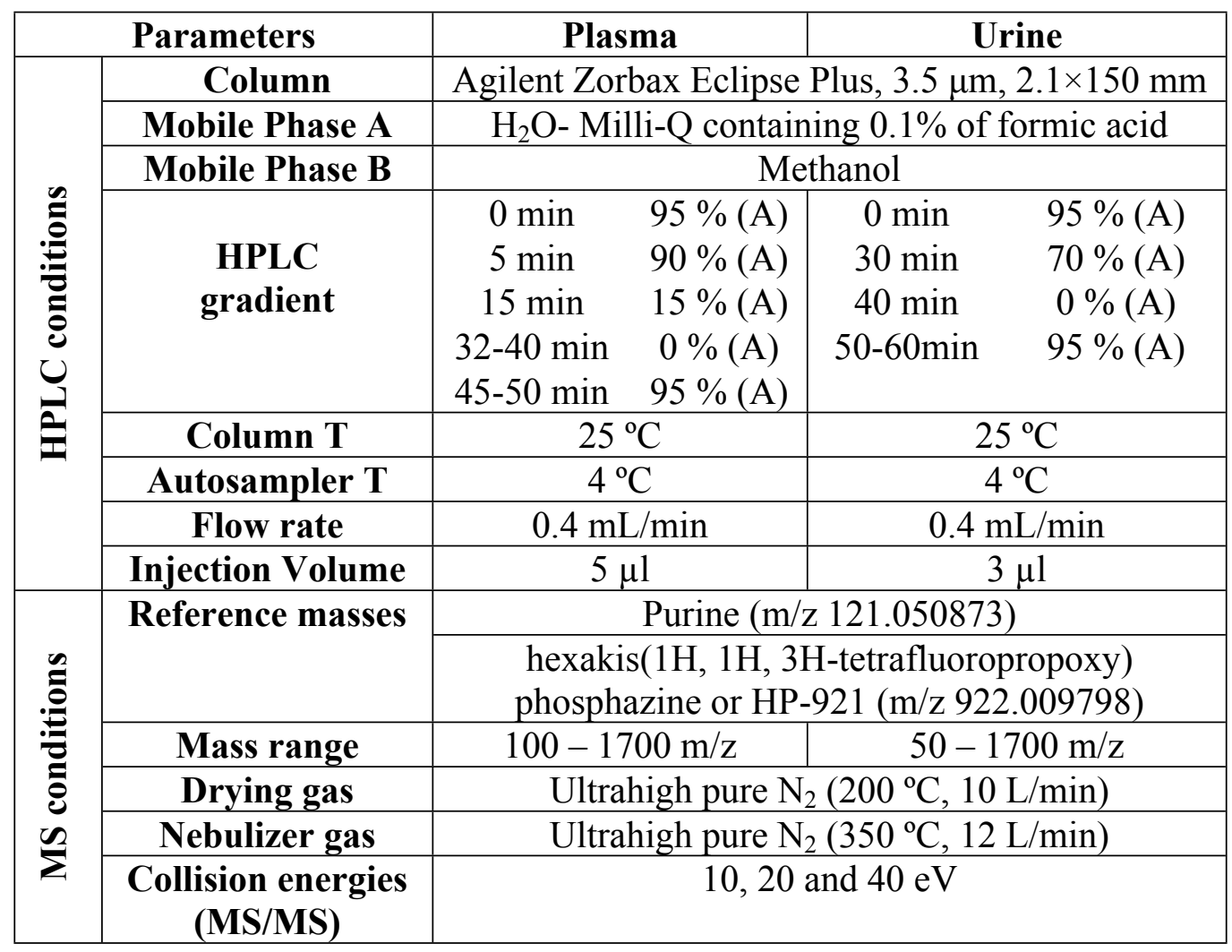


Table S3. Data processing parameters optimized by IPO

Table S3. Parameters optimized by IPO that were used for peak peaking, alignment and grouping carried out with the XCMS package. Parameters optimized for RamClustR package. *These parameters were fixed and therefore, not optimized by IPO.

\begin{tabular}{|c|c|c|c|}
\hline \multicolumn{2}{|c|}{ Parameters } & Urine & Plasma \\
\hline \multirow{7}{*}{ 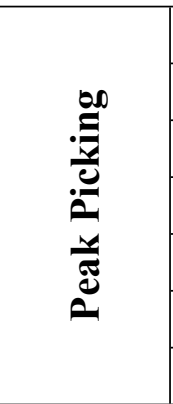 } & method & centwave & centwave \\
\hline & min_peakwidth & 11.2 & 12.45 \\
\hline & max_peakwidth & 48.0 & 35.0 \\
\hline & ppm & 29.8 & 24.0 \\
\hline & mzdiff & 0.00725 & 0.00175 \\
\hline & noise* & 1000 & 1000 \\
\hline & prefilter* & $(3,800)$ & $(3,800)$ \\
\hline \multirow{5}{*}{ 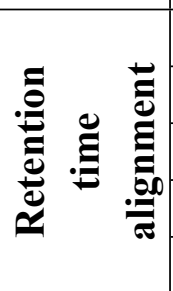 } & method & obiwarp & obiwarp \\
\hline & profStep & 1.00 & 0.30 \\
\hline & response & 4.42 & 13.84 \\
\hline & gapInit & 0.768 & 0.352 \\
\hline & gapExtend & 2.98 & 2.44 \\
\hline \multirow{4}{*}{ } & method & density & density \\
\hline & bw & 5.48 & 5.00 \\
\hline & minfrac* & 0.5 & 0.5 \\
\hline & mzwid & 0.033 & 0.047 \\
\hline \multirow{2}{*}{ 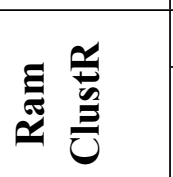 } & $\sigma_{t^{*}}$ & 2.50 & 1.33 \\
\hline & $\sigma_{\mathrm{r}^{*}}$ & 0.3 & 0.3 \\
\hline
\end{tabular}


Table S4. List of metabolites selected in the MUVR-PLS models that compared all cases versus HC

List of metabolites selected in the MUVR-PLS models that compared all cases versus healthy controls (FC $>0$, metabolites overexpressed in SAD cases).

\begin{tabular}{|c|c|c|c|c|c|c|c|c|c|c|}
\hline & $\begin{array}{c}\text { Mass } \\
\text { (Da) }\end{array}$ & $\mathrm{RT}(\min )$ & Rank & FC & FDR & $\begin{array}{l}\text { Molecular } \\
\text { Formula }\end{array}$ & $\begin{array}{c}\text { Score } \\
(\%)\end{array}$ & Compound Name & MS/MS Fragments & $\begin{array}{c}\text { Identification } \\
\text { Database }\end{array}$ \\
\hline \multirow{11}{*}{ 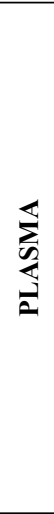 } & 208.0848 & 4.3 & 201.67 & 1.29 & $3.41 \mathrm{E}-3$ & $\mathrm{C}_{10} \mathrm{H}_{12} \mathrm{~N}_{2} \mathrm{O}_{3}$ & 85.66 & L-kynurenine & $74.0216 / 94.0627 / 120.0413 / 146.0561$ & HMDB00684 \\
\hline & 184.1212 & 9.0 & 13.81 & 1.51 & $2.58 \mathrm{E}-4$ & $\mathrm{C}_{9} \mathrm{H}_{16} \mathrm{~N}_{2} \mathrm{O}_{2}$ & 86.53 & N-(3-acetamidopropyl)pyrrolidin-2-one & $56.9408 / 86.9471 / 98.0591 / 126.0905 / 185.1262$ & HMDB61384 \\
\hline & 412.1325 & 17.8 & 17.76 & -2.07 & $3.72 \mathrm{E}-4$ & $\mathrm{C}_{15} \mathrm{H}_{20} \mathrm{~N}_{6} \mathrm{O}_{8}$ & 84.47 & N6-Carbamoyl-L-threonyladenosine & $136.0602 / 162.0392 / 281.09852 / 413.1398$ & Metlin95993 \\
\hline & 328.2363 & 24.4 & 48.68 & 1.95 & $2.70 \mathrm{E}-3$ & $\mathrm{C}_{22} \mathrm{H}_{32} \mathrm{O}_{2}$ & 83.41 & Docosahexanoic acid & $107.0814 / 145.0975 / 173.1304 / 161.1285$ & HMDB02183 \\
\hline & 319.1911 & 24.65 & 21.53 & 1.41 & $4.88 \mathrm{E}-3$ & ------ & ------ & Unknown & -------- & ------- \\
\hline & 278.2193 & 25.7 & 9.29 & 1.74 & $9.92 \mathrm{E}-4$ & $\mathrm{C}_{18} \mathrm{H}_{30} \mathrm{O}_{2}$ & 87.00 & Linolenic Acid & 69.0660/95.0833/123.1134/279.2177 & HMDB01388 \\
\hline & 304.2351 & 26.4 & 8.90 & 1.53 & $2.58 \mathrm{E}-4$ & $\mathrm{C}_{20} \mathrm{H}_{32} \mathrm{O}_{2}$ & 87.11 & Arachidonic acid & $\begin{array}{c}55.0530 / 57.0685 / 67.0526 / 71.0842 / \\
107.0811 / 121.0995 / 123.0117 / 161.112 / 177.3601 \\
\end{array}$ & HMDB01043 \\
\hline & 676.4568 & 26.4 & 6.28 & 1.59 & $3.72 \mathrm{E}-4$ & $\mathrm{C}_{36} \mathrm{H}_{69} \mathrm{O}_{9} \mathrm{P}$ & 97.2 & $\mathrm{PG}(\mathrm{P}-30: 1)$ & 699.4461 (Level 3) & LMGP04030004 \\
\hline & 306.2498 & 28.5 & 38.48 & 1.57 & $1.21 \mathrm{E}-3$ & $\mathrm{C}_{20} \mathrm{H}_{34} \mathrm{O}_{2}$ & 83.9 & Sciadonic acid & $55.0524 / 95.0155$ & HMDB31058 \\
\hline & 363.2483 & 28.5 & 59.41 & 1.30 & 0.041 & ----- & $\begin{array}{ll}----- \\
\end{array}$ & Unknown & -------- & ------- \\
\hline & 780.6045 & 38.7 & 72.17 & -1.11 & $<0.05$ & $\mathrm{C}_{45} \mathrm{H}_{85} \mathrm{~N}_{2} \mathrm{O}_{6} \mathrm{P}$ & 94.43 & C22:3 Sphingomyelin & $86.0939 / 184.0699 / 598.5429$ & HMDB13468 \\
\hline \multirow{4}{*}{ 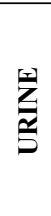 } & 277.1162 & 1.3 & 8.95 & -1.47 & $2.94 \mathrm{E}-3$ & $\mathrm{C}_{11} \mathrm{H}_{19} \mathrm{NO}_{7}$ & 84.57 & N-(1-Deoxy-1-fructosyl)proline & $100.0755 / 130.0494 / 214.1060 / 232.1174$ & HMDB38493 \\
\hline & 253.0885 & 1.4 & 283.05 & 1.71 & $1.67 \mathrm{E}-3$ & ----- & $\begin{array}{ll}----- \\
\end{array}$ & Unknown & -------- & ------- \\
\hline & 210.0642 & 6.6 & 15.23 & 2.17 & 0.011 & $\mathrm{C}_{9} \mathrm{H}_{10} \mathrm{~N}_{2} \mathrm{O}_{4}$ & 83.17 & N-(5amino)-2-hydroxybenzoylglycine & $108.0449 / 109.0526 / 150.0418 / 165.0648$ & HMDB61683 \\
\hline & 169.5949 & 13.86 & 165.05 & 1.53 & 0.025 & ------ & ------ & Unknown & $\begin{array}{l}------- \\
\end{array}$ & ------- \\
\hline
\end{tabular}




\section{Table S5. Unknown compounds}

List of the parameters $(\mathrm{m} / \mathrm{z}, \mathrm{RT}$, and ranks) of the unknown compounds selected from the MUVR-PLS models obtained from plasma and urine from individual SADs and healthy controls.

\begin{tabular}{|c|c|c|c|c|c|}
\hline \multicolumn{3}{|c|}{ Unknowns (Plasma samples) } & \multicolumn{3}{|c|}{ Unknowns (Urine samples) } \\
\hline $\mathrm{m} / \mathrm{z}$ & RT (min) & Diseases (Rank) & $\mathrm{m} / \mathrm{z}$ & RT (min) & Diseases (Rank) \\
\hline 175.0341 & 10.64 & SJS (109.8) & 157.0609 & 1.04 & $\operatorname{SSC}(71.6), \mathrm{RA}(258.9)$ \\
\hline 216.0664 & 12.67 & $\operatorname{RA}(144.1)$ & 187.0585 & 1.10 & $\mathrm{RA}(40.1)$ \\
\hline 199.1309 & 13.04 & $\mathrm{RA}(74.6)$ & 278.1235 & 1.32 & $\operatorname{SSC}(9.3), \operatorname{SLE}(36.5), \operatorname{SJS}(26.9)$ \\
\hline 601.2651 & 14.39 & SJS (139.3) & 142.0862 & 1.37 & $\mathrm{RA}(187.4)$ \\
\hline 171.1488 & 14.73 & SJS (103.4), SLE(151.2) & 254.0885 & 1.38 & $\operatorname{RA}(25.9), \operatorname{SSC}(28.9), \operatorname{SJS}(33.5)$ \\
\hline 268.0597 & 15.2 & RA(127.5), SLE(114.5) & 226.0826 & 1.48 & $\operatorname{SLE}(5.7), \operatorname{SJS}(16.1)$ \\
\hline 374.1953 & 15.20 & $\mathrm{RA}(118.4)$ & 241.1553 & 1.52 & SJS(204.7) \\
\hline 475.3245 & 16.08 & $\operatorname{RA}(129.4)$ & 150.0186 & 1.63 & $\operatorname{ssc}(184.5)$ \\
\hline 276.9894 & 16.17 & $\operatorname{SJS}(82.3), \operatorname{RA}(79.5)$ & 305.0978 & 2.35 & $\operatorname{sSC}(38.1)$ \\
\hline 398.2388 & 17.27 & SLE(139.9) & 238.0934 & 2.54 & $\operatorname{RA}(38.5)$ \\
\hline 415.2777 & 20.47 & SJS (31.1) & 273.1268 & 6.95 & $\operatorname{SLE}(52.9)$ \\
\hline 526.2871 & 21.69 & SJS (155.2) & 159.5907 & 13.89 & $\operatorname{SLE}(30.6)$ \\
\hline 619.4329 & 22.80 & SJS (137.8) & 146.5826 & 14.02 & $\operatorname{SLE}(79.7)$ \\
\hline 680.3053 & 23.07 & SJS (162.0) & 512.2218 & 16.62 & $\operatorname{SLE}(85.8)$ \\
\hline 303.2235 & 24.64 & $\operatorname{SLE}(73.5)$ & 322.1315 & 18.35 & $\operatorname{SLE}(29.7)$ \\
\hline 319.1910 & 24.65 & $\operatorname{SJS}(50.8), \operatorname{SSC}(29.2), \operatorname{SLE}(27.4)$ & 276.1262 & 19.90 & $\operatorname{SLE}(42.5)$ \\
\hline 461.1812 & 24.66 & $\operatorname{SJS}(42.4)$ & 175.5557 & 19.91 & $\operatorname{SLE}(39.4)$ \\
\hline 396.2137 & 26.35 & SJS (198.1) & 366.1577 & 25.55 & SLE(98.3) \\
\hline 364.2483 & 28.49 & SJS (8.8) & 484.1482 & 27.44 & $\operatorname{SLE}(81.0)$ \\
\hline 617.4690 & 28.74 & SJS (141.1) & 350.1628 & 30.21 & $\operatorname{SLE}(10.7)$ \\
\hline 769.5841 & 36.50 & SJS (124.2) & 332.2433 & 34.61 & $\operatorname{sSC}(43.5)$ \\
\hline 806.0530 & 35.59 & SJS (112.8) & 277.1412 & 39.36 & SJS(166.3), RA(44.9) \\
\hline \multirow[t]{8}{*}{643.5204} & 41.24 & SJS (176.5) & 250.1186 & 40.15 & SLE(204.5) \\
\hline & & & 219.1744 & 40.56 & $\operatorname{SLE}(217.7)$ \\
\hline & & & 535.2877 & 41.00 & $\operatorname{RA}(11.2), \operatorname{SJS}(38.0)$ \\
\hline & & & 318.2406 & 41.85 & $\mathrm{RA}(31.9), \mathrm{UCTD}()$ \\
\hline & & & 269.2094 & 42.02 & $\operatorname{SSC}(91.1)$ \\
\hline & & & 640.5906 & 43.38 & $\operatorname{sJS}(29.5)$ \\
\hline & & & 522.5991 & 43.49 & $\mathrm{RA}(92.3)$ \\
\hline & & & 282.2800 & 43.99 & $\operatorname{SsC}(52.9), \operatorname{SJS}(36.0)$ \\
\hline
\end{tabular}


Table S6. ANOVA results for the top six metabolites

ANOVA results for the top six metabolites selected in a larger number of PLS models.

\begin{tabular}{|c|c|c|}
\hline Metabolite & FDR (ANOVA) & Post-hoc tests \\
\hline Docosahexanoic acid & $6.28 \mathrm{E}-3$ & MCTD-HC, RA-HC, SJS-HC, SLE-HC, SSC-HC, UCTD-HC \\
\hline Linolenic Acid & $2.74 \mathrm{E}-3$ & MCTD-HC, RA-HC, SJS-HC, SLE-HC, SSC-HC, UCTD-HC \\
\hline Stearidonic acid & 0.010 & RA-HC, SJS-HC, SLE-HC, SSC-HC, UCTD-HC \\
\hline N-(3-acetamidopropyl)pyrrolidin-2-one & $1.63 \mathrm{E}-4$ & RA-HC, SJS-HC, SLE-HC, SSC-HC, UCTD-HC, RA-MCTD, SLE-MCTD, \\
SSC-MCTD, UCTD-MCTD \\
\hline N6-Carbamoyl-L-threonyladenosine & 2.74 E-3 & HC-MCTD, HC-RA, HC-SJS. HC-SLE. HC-SSC. HC-UCTD \\
\hline N-(5amino)-2-hydroxybenzoylglycine & $7.10 \mathrm{E}-3$ & PAPS-HC, RA-HC, SJS-HC, SLE-HC, SSC-HC, SLE-MCTD \\
\hline
\end{tabular}


Table S7. Annotated metabolites selected from the PLS models (individual SADs versus UCTD)

\begin{tabular}{|c|c|c|c|c|c|c|c|c|}
\hline \multirow{11}{*}{$\frac{\pi}{\frac{\pi}{4}}$} & $\begin{array}{l}\text { Mass } \\
(\mathrm{Da})\end{array}$ & $\begin{array}{c}\text { RT } \\
\text { (min) }\end{array}$ & $\begin{array}{c}\text { Diseases } \\
\text { (Rank) }\end{array}$ & $\begin{array}{c}\text { Molecular } \\
\text { Formula }\end{array}$ & $\begin{array}{c}\text { Score } \\
(\%)\end{array}$ & Compound Name & MS/MS Fragments & $\begin{array}{c}\text { Identification } \\
\text { Database }\end{array}$ \\
\hline & 159.0527 & 1.1 & $\operatorname{MCTD}(8.1)$ & $\mathrm{C}_{6} \mathrm{H}_{9} \mathrm{NO}_{4}$ & 96.77 & N-Methyl-2-oxoglutaramate & $56.0480 / 74.0584 / 86.0586 / 114.0527$ & $\mathrm{C} 03623$ \\
\hline & 112.0271 & 2.1 & $\operatorname{MCTD}(18.6)$ & --- & --- & Unknown & ---- & ---- \\
\hline & 164.0498 & 2.4 & $\mathrm{RA}(20.6), \operatorname{MCTD}(71.9)$ & $\mathrm{C}_{9} \mathrm{H}_{8} \mathrm{O}_{3}$ & 98.94 & m-Coumaric acid & $77.0370 / 91.0522 / 119.0469 / 147.0410$ & HMDB62774 \\
\hline & 208.0848 & 4.3 & $\operatorname{SSC}(49.3)$ & $\mathrm{C}_{10} \mathrm{H}_{12} \mathrm{~N}_{2} \mathrm{O}_{3}$ & 85.66 & L-kynurenine & $74.0216 / 94.0627 / 120.0413 / 146.0561$ & HMDB00684 \\
\hline & 156.0763 & 8.1 & $\operatorname{SSC}(11.9)$ & ---- & --- & Unknown & ---- & ---- \\
\hline & 287.1204 & 15.2 & $\mathrm{RA}(100.2)$ & $\mathrm{C}_{15} \mathrm{H}_{29} \mathrm{NO}_{4}$ & 93.89 & L-Octanoylcarnitine & $60.0785 / 85.0262 / 310.1934$ & HMDB00834 \\
\hline & 495.3308 & 21.9 & $\mathrm{RA}(80.8)$ & $\mathrm{C}_{24} \mathrm{H}_{50} \mathrm{NO}_{7} \mathrm{P}$ & 90.62 & LysoPC(16:0) & $86.0953 / 104.1060 / 146.9804 / 459.2470$ & HMDB10382 \\
\hline & 569.3426 & 22.3 & $\operatorname{MCTD}(32.2)$ & $\mathrm{C}_{30} \mathrm{H}_{52} \mathrm{NO}_{7} \mathrm{P}$ & 68.90 & LysoPC(22:5) & $86.0954 / 104.1055 / 146.9802 / 483.2450 / 533.2598$ & HMDB10403 \\
\hline & 481.3109 & 24.9 & $\operatorname{MCTD}(24.7)$ & $\mathrm{C}_{23} \mathrm{H}_{48} \mathrm{NO}_{7} \mathrm{P}$ & 71.25 & LysoPC(15:0) & $482.3182($ level3) & HMDB10381 \\
\hline & 616.4617 & 28.7 & $\operatorname{SSC}(31.6)$ & ---- & ---- & Unknown & ---- & ----- \\
\hline \multirow{20}{*}{$\frac{\frac{u}{z}}{\frac{\alpha}{\supset}}$} & 113.0596 & 1.0 & $\operatorname{SJS}(260.5)$ & $\mathrm{C}_{4} \mathrm{H}_{7} \mathrm{~N}_{3} \mathrm{O}$ & 97.2 & Creatinine & $43.0287 / 44.0492 / 58.0651 / 86.0810$ & HMDB00562 \\
\hline & 112.0274 & 1.2 & $\operatorname{SLE}(44.5), \operatorname{SSC}(33.7)$ & $\mathrm{C}_{4} \mathrm{H}_{4} \mathrm{~N}_{2} \mathrm{O}_{2}$ & 86.3 & Uracil & 113.0350 (level 3) & HMDB00300 \\
\hline & 225.0753 & 1.5 & SLE(147.7) & ---- & ---- & Unknown & ---- & ---- \\
\hline & 240.1480 & 1.5 & $\operatorname{SJS}(226.9)$ & ---- & --- & Unknown & ---- & ----- \\
\hline & 244.0669 & 1.5 & $\operatorname{SJS}(344.2)$ & $\mathrm{C}_{9} \mathrm{H}_{12} \mathrm{~N}_{2} \mathrm{O}_{6}$ & 93.7 & Uridine & $82.0291 / 125.0348 / 139.0503 / 155.0450 / 209.0552$ & HMDB00296 \\
\hline & 136.0338 & 1.9 & $\operatorname{SJS}(89.2), \operatorname{SSC}(56.6)$ & $\mathrm{C}_{5} \mathrm{H}_{4} \mathrm{~N}_{4} \mathrm{O}$ & 95.33 & Hypoxanthine & $55.0285 / 67.0289 / 82.0395 / 94.0398 / 110.0351 / 119.0353$ & HMDB00157 \\
\hline & 304.0905 & 2.4 & $\operatorname{SSC}(66.9), \operatorname{SJS}(92.1)$ & $\mathrm{C}_{11} \mathrm{H}_{16} \mathrm{~N}_{2} \mathrm{O}_{8}$ & 84.21 & N-Acetylaspartylglutamic acid & $305.0978($ level3) & HMDB01067 \\
\hline & 228.1478 & 2.9 & $\operatorname{SJS}(351.9)$ & $\mathrm{C}_{11} \mathrm{H}_{20} \mathrm{~N}_{2} \mathrm{O}_{3}$ & 97.89 & L-isoleucyl-L-proline & $60.0806 / 70.0653 / 114.0552$ & HMDB11174 \\
\hline & 198.0706 & 5.5 & $\operatorname{SLE}(242.0)$ & ---- & --- & Unknown & ----- & ---- \\
\hline & 99.0683 & 6.6 & SLE(443.6) & $\mathrm{C}_{5} \mathrm{H}_{9} \mathrm{NO}$ & 87.19 & 2-Piperidinone & $44.0127 / 56.0487$ & HMDB11749 \\
\hline & 297.1074 & 7.1 & $\operatorname{SJS}(182.4)$ & $\mathrm{C}_{11} \mathrm{H}_{15} \mathrm{~N}_{5} \mathrm{O}_{5}$ & 97.5 & Methylguanosine & $109.0506 / 110.0350 / 135.0300 / 149.0461 / 166.0724 / 167.0750$ & HMDB01563 \\
\hline & 150.0543 & 7.3 & $\operatorname{SJS}(305.2)$ & $\mathrm{C}_{6} \mathrm{H}_{6} \mathrm{~N}_{4} \mathrm{O}$ & 96.87 & 1-Methylhypoxanthine & $42.0332 / 55.0283 / 82.0397 / 94.0394 / 110.0347$ & HMDB13141 \\
\hline & 303.1567 & 8.8 & $\operatorname{SJS}(77.7)$ & $\mathrm{C}_{16} \mathrm{H}_{21} \mathrm{~N}_{3} \mathrm{O}_{3}$ & 84.35 & Tryptophyl-Valine & $124.0497 / 149.0440 / 179.0789 / 194.1010$ & HMDB29096 \\
\hline & 311.1233 & 11.0 & $\operatorname{SJS}(87.8)$ & $\mathrm{C}_{12} \mathrm{H}_{17} \mathrm{~N}_{5} \mathrm{O}_{5}$ & 95.32 & 1,7-Dimethylguanosine & 180.0760 & HMDB01961 \\
\hline & 383.1078 & 12.7 & $\operatorname{SJS}(248.6)$ & $\mathrm{C}_{14} \mathrm{H}_{17} \mathrm{~N}_{5} \mathrm{O}_{8}$ & 98.76 & Succinyladenosine & $136.0611 / 192.0512 / 234.0620 / 252.0756$ & HMDB00912 \\
\hline & 512.2145 & 16.4 & $\operatorname{SSC}(95.5)$ & ---- & ---- & Unknown & ----- & ----- \\
\hline & 349.1562 & 19.9 & $\operatorname{SSC}(115.9)$ & ---- & ---- & Unknown & ---- & ---- \\
\hline & 412.1344 & 22.1 & $\operatorname{SJS}(114.1)$ & $\mathrm{C}_{15} \mathrm{H}_{20} \mathrm{~N}_{6} \mathrm{O}_{8}$ & 96.42 & N6-Carbamoyl-L-threonyladenosine & $136.0602 / 162.0392 / 281.09852$ & HMDB41623 \\
\hline & 116.5186 & 40.5 & $\operatorname{SLE}(33.7)$ & ---- & --- & Unknown & ---- & ---- \\
\hline & 317.2333 & 41.9 & SLE(492.46) & ---- & $\begin{array}{c}--- \\
\end{array}$ & Unknown & $\begin{array}{ll}---- \\
\end{array}$ & $\begin{array}{ll}---- \\
-1\end{array}$ \\
\hline
\end{tabular}


Figure S1. PCA scores plots before and after normalization (color code by batch)

PCA scores plots from data before (1a. Plasma; 1b, Urine) and after (1c: Plasma; 1d: Urine) batchCorr normalization. (color code by analytical batches).

a)

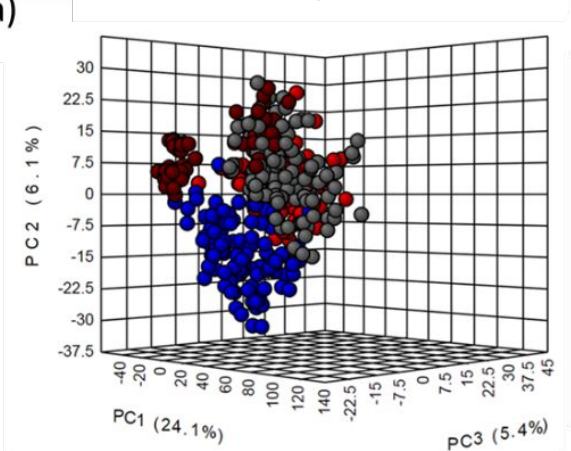

b)

URINE DATA c)

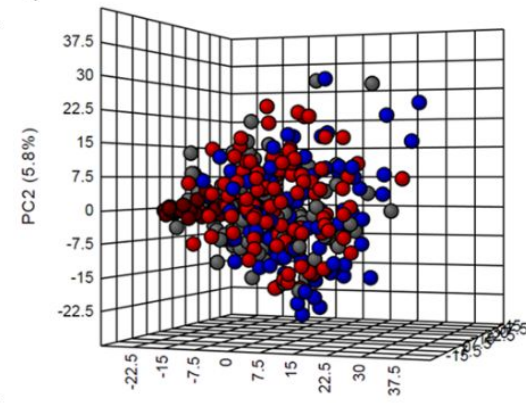

Normalized

d)

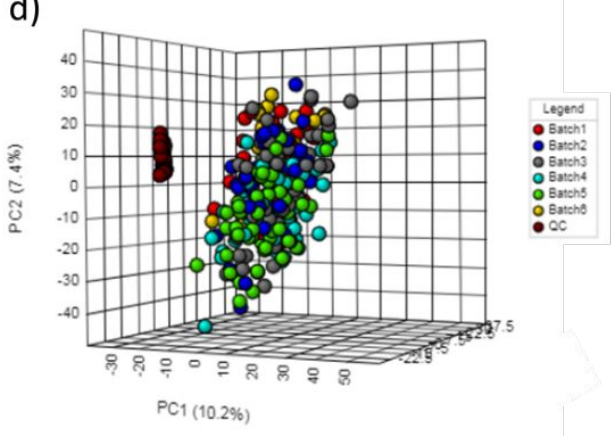


Figure S2. PCA scores plots before and after normalization (color by class)

PCA scores plots of the normalized data of plasma (a) and urine data (b). (Color code by sample categories).

a) plasma

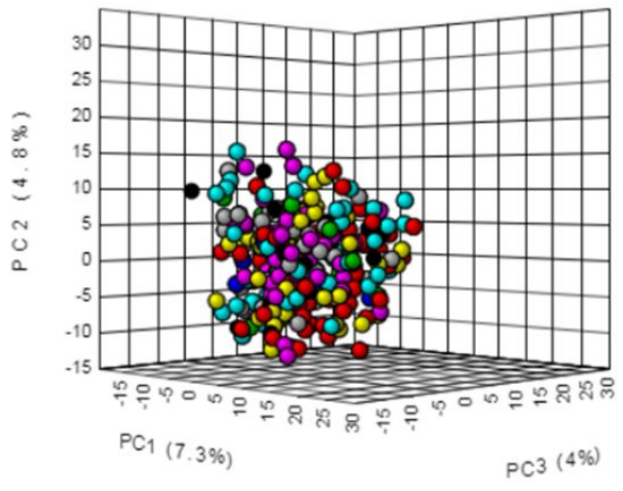

b) urine

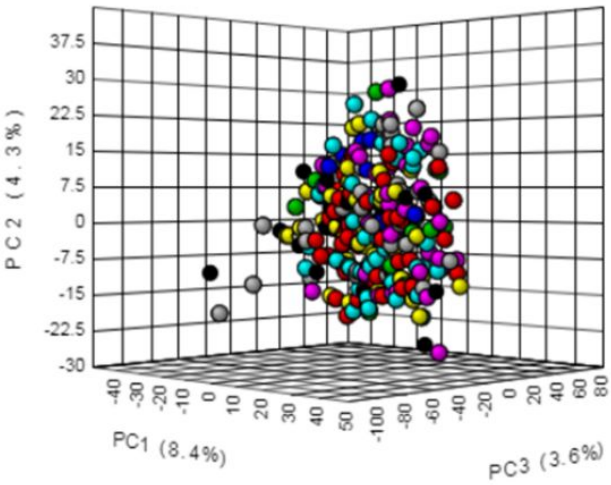

Figure S3. Results of the MUVR-PLS models of specific SADs versus HC.

Confusion matrices, percentages of well-classified samples and percentages of samples classified as SAD obtained in the PLS models created with the metabolomic data of four SADs (RA, SjS, SLE and SSC) and HC.

\begin{tabular}{|c|c|c|c|c|c|}
\hline Plasn & $\mathrm{a} \mathrm{m}$ & del & & pred & cted \\
\hline & $\mathrm{HC}$ & RA & SjS & SLE & SSC \\
\hline $\mathrm{HC}$ & 48 & 4 & 0 & 0 & 3 \\
\hline RA & 14 & 16 & 3 & 2 & 12 \\
\hline SJS & 10 & 7 & 4 & 13 & 12 \\
\hline SLE & 12 & 5 & 0 & 24 & 5 \\
\hline SSC & 10 & 9 & 0 & 3 & 21 \\
\hline
\end{tabular}

\begin{tabular}{|c|}
\hline $\begin{array}{c}\text { Well classified } \\
\text { samples (\%) }\end{array}$ \\
\hline 87.3 \\
\hline 34.0 \\
\hline 8.7 \\
\hline 52.2 \\
48.9 \\
\hline
\end{tabular}

Samples classified as SAD (\%)

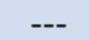

70.2

78.3

73.9

76.7

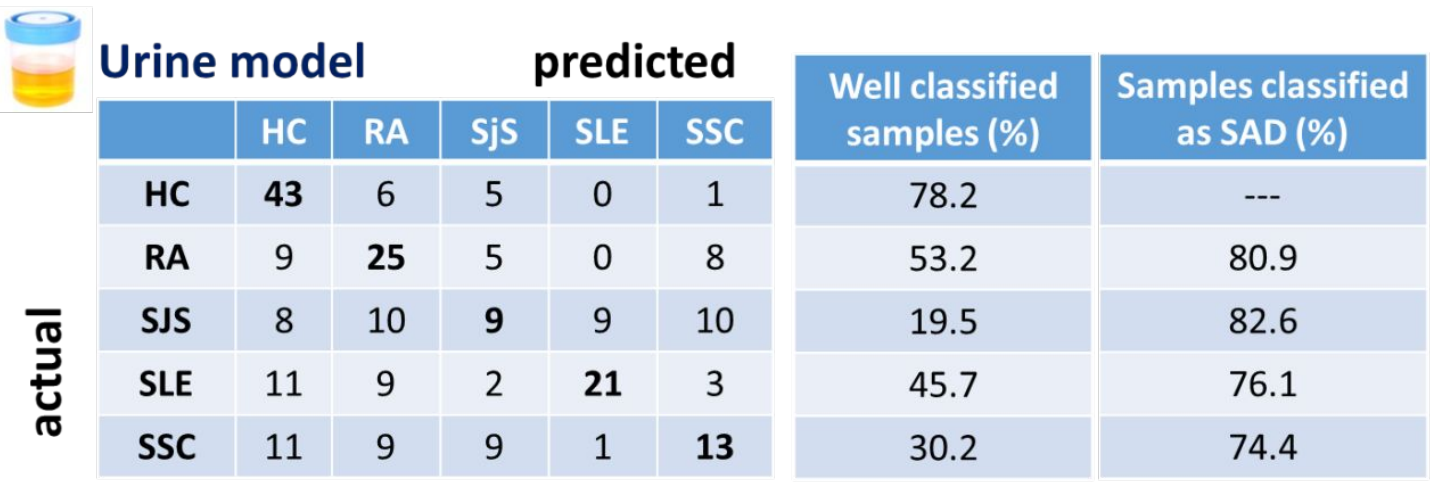




\section{Author contribution}

A.F-O performed the sample treatments and analysis as well as the data treatment and wrote the manuscript. C.B contributed with statistical analysis, results discussion and writing of the manuscript. I.B-L and R.Q-P contributed with the mass spectrometry analysis. M.d.1.L.C.-G participated in the biological interpretation of the results. A.S-C and M.E.A designed and supervised the study.

\section{Acknowledgments}

Biological samples used in this study were obtained from the Precisesads Clinical Consortium. As such, the investigators within this consortium were responsible for the recruitment of volunteers, carrying out the collection of biological samples and their distribution to the metabolomics laboratory. Furthermore, they provided the clinical data of the volunteers but did not participate in the analysis or writing of this manuscript. The contribution of the clinicians who provided the biological samples and clinical data is recognized by including their detailed names responsible for this consortium in an appendix list "PRECISESADS Clinical Consortium" in the Supporting Information. The main objective of the PRECISESADS project (www.precisesads.eu) is to reclassify the individuals affected by SADs into molecular clusters instead of clinical entities through the determination of molecular profiles using several "-omics" techniques. This European project has received support from the Innovative Medicines Initiative Joint Undertaking under grant agreement $\mathrm{n}^{\circ} 115565$, resources of which are composed of financial contribution from the European Union's Seventh Framework Programme (FP7/2007-2013) and EFPIA companies' in kind contribution. 